\title{
On Free Field Realizations of $W(2,2)$-Modules
}

\author{
Dražen ADAMOVIĆ $\dagger$ and Gordan RADOBOLJA \\ $\dagger$ Department of Mathematics, University of Zagreb, Bijenička 30, 10000 Zagreb, Croatia \\ E-mail: adamovic@math.hr \\ URL: https://web.math.pmf .unizg.hr/ adamovic/ \\ $¥$ Faculty of Science, University of Split, Rudera Boškovića 33, 21000 Split, Croatia \\ E-mail: gordan@pmfst.hr
}

Received June 09, 2016, in final form December 03, 2016; Published online December 06, 2016

http://dx.doi.org/10.3842/SIGMA.2016.113

\begin{abstract}
The aim of the paper is to study modules for the twisted Heisenberg-Virasoro algebra $\mathcal{H}$ at level zero as modules for the $W(2,2)$-algebra by using construction from $[J$. Pure Appl. Algebra 219 (2015), 4322-4342, arXiv:1405.1707]. We prove that the irreducible highest weight $\mathcal{H}$-module is irreducible as $W(2,2)$-module if and only if it has a typical highest weight. Finally, we construct a screening operator acting on the Heisenberg-Virasoro vertex algebra whose kernel is exactly $W(2,2)$ vertex algebra.
\end{abstract}

Key words: Heisenberg-Virasoro Lie algebra; vertex algebra; $W(2,2)$ algebra; screeningoperators

2010 Mathematics Subject Classification: 17B69; 17B67; 17B68; 81R10

\section{Introduction}

Lie algebra $W(2,2)$ was first introduced by W. Zhang and C. Dong in [20] as part of a classification of certain simple vertex operator algebras. Its representation theory has been studied in $[14,15,18,19]$ and several other papers. Although $W(2,2)$ is an extension of the Virasoro algebra, its representation theory is very different. This is most notable with highest weight representations. It was shown in [19] that some Verma modules contain a cosingular vector.

Highest weight representation theory of the twisted Heisenberg-Virasoro Lie algebra has also been studied recently. Representations with nontrivial action of $C_{I}$ have been developed in [6]. Representations at level zero, i.e., with trivial action of $C_{I}$ were studied in [8] due to their importance in some constructions over the toroidal Lie algebras (see [7,9]). In this case, a free field realization of highest weight modules along with the fusion rules for a suitable category of modules were obtained in [4].

Irreducible highest weight modules of highest weights $(0,0)$ over these algebras carry the structure of simple vertex operator algebras. Let us denote these vertex operator algebras as $L^{W(2,2)}\left(c_{L}, c_{W}\right)$ and $L^{\mathcal{H}}\left(c_{L}, c_{L, I}\right)$. It was proved in [4] that simple vertex operator algebra $L^{W(2,2)}\left(c_{L}, c_{W}\right)$ embeds into Heisenberg-Virasoro vertex operator algebra $L^{\mathcal{H}}\left(c_{L}, c_{L, I}\right)$ so that $c_{W}=-24 c_{L, I}^{2}$. As a result each highest weight module over $\mathcal{H}$ is also a $W(2,2)$-module. In this paper we shall completely describe the structure of the irreducible highest weight $\mathcal{H}$ modules as $W(2,2)$-modules. We show that in generic case the resulting $W(2,2)$-module is irreducible. However, in case of a module of highest weight such that associated Verma module over $W(2,2)$ contains cosingular vectors (we shall call this kind of weight atypical), irreducible $\mathcal{H}$-module is reducible over $W(2,2)$. We shall denote the irreducible highest weight $\mathcal{H}$-module 
$L^{\mathcal{H}}\left(c_{L}, 0, c_{L, I}, h, h_{I}\right)$ shortly as $L^{\mathcal{H}}\left(h, h_{I}\right)$. We also use the following notation ${ }^{1}$

$$
h_{p, r}=\left(1-p^{2}\right) \frac{c_{L}-2}{24}+p(p-1)+p \frac{1-r}{2}
$$

for $p, r \in \mathbb{Z}_{>0}$. Define

$$
\mathcal{A T}_{\mathcal{H}}\left(c_{L}, c_{L, I}\right)=\left\{\left(h_{p, r},(1 \pm p) c_{L, I}\right) \mid p, r \in \mathbb{Z}_{>0}\right\} .
$$

We call a weight $\left(h, h_{I}\right)$ atypical for $\mathcal{H}$ (resp. typical) if $\left(h, h_{I}\right) \in \mathcal{A T}_{\mathcal{H}}\left(c_{L}, c_{L, I}\right)$ (resp. $\left(h, h_{I}\right) \notin$ $\left.\mathcal{A} \mathcal{T}_{\mathcal{H}}\left(c_{L}, c_{L, I}\right)\right)$. We shall refer to a highest weight module over $\mathcal{H}$ as (a)typical if its highest weight is (a)typical for $\mathcal{H}$.

The next theorem gives a main result of the paper.

Theorem 1.1. Assume that $c_{L, I} \neq 0$.

(1) $L^{\mathcal{H}}\left(h, h_{I}\right)$ is irreducible as a $W(2,2)$-module if and only if

$$
\left(h, h_{I}\right) \notin \mathcal{A} \mathcal{T}_{\mathcal{H}}\left(c_{L}, c_{L, I}\right) .
$$

(2) If $\left(h, h_{I}\right) \in \mathcal{A T}_{\mathcal{H}}\left(c_{L}, c_{L, I}\right)$ then $L^{\mathcal{H}}\left(h, h_{I}\right)$ is a non-split extension of two irreducible highest weight $W(2,2)$-modules.

We recall some aspects of representation theories of infinite-dimensional Lie algebras $\mathcal{H}$ and $W(2,2)$ in Section 2. The main results on the branching rules will be proved in Section 3. From the free field realization in [4] follows that irreducible $\mathcal{H}$-modules are pairwise contragredient. For half of these modules, proofs rely on a $W(2,2)$-homomorphism between Verma modules over $W(2,2)$ and $\mathcal{H}$ which is induced by a homomorphism of vertex operator algebras. The rest is then proved elegantly by passing to contragredients. We also prove a very interesting result that the Verma module for $\mathcal{H}$ with typical highest weight is an infinite direct sum of irreducible $W(2,2)$-modules (cf. Theorem 3.7). This result presents a $W(2,2)$-analogue of certain Feigin-Fuchs modules for the Virasoro algebra (cf. Remark 3.8).

From the results in the paper, we see that the vertex algebra $L^{W(2,2)}\left(c_{L}, c_{W}\right)$ has many properties similar to the $\mathcal{W}$-algebras appearing in logarithmic conformal field theory (LCFT):

- $L^{W(2,2)}\left(c_{L}, c_{W}\right)$ admits a free field realization inside of the Heisenberg-Virasoro vertex algebra $L^{\mathcal{H}}\left(c_{L}, c_{L, I}\right)$.

- Typical modules are realized as irreducible modules for $L^{\mathcal{H}}\left(c_{L}, c_{L, I}\right)$.

- In the atypical case, irreducible $L^{\mathcal{H}}\left(c_{L}, c_{L, I}\right)$-modules as $L^{W(2,2)}\left(c_{L}, c_{W}\right)$-modules have semi-simple rank two.

The singlet vertex algebra $\overline{M(1)}$ has similar properties. $\overline{M(1)}$ is realized as kernel of a screening operator inside the Heisenberg vertex algebra $M(1)$ (cf. [1]). In Section 4 we construct the screening operator

$$
S_{1}: L^{\mathcal{H}}\left(c_{L}, c_{L, I}\right) \rightarrow L^{\mathcal{H}}(1,0),
$$

which commutes with the action of $W(2,2)$-algebra such that

$$
\operatorname{Ker}_{L^{\mathcal{H}}\left(c_{L}, c_{L, I}\right)} S_{1} \cong L^{W(2,2)}\left(c_{L}, c_{W}\right) .
$$

Our construction uses an extension $\mathcal{V}_{\text {ext }}$ of the vertex algebra $L^{\mathcal{H}}\left(c_{L}, c_{L, I}\right)$ by a non-weight module for the Heisenberg-Virasoro vertex algebra. In our forthcoming paper [5], we shall present an explicit realization of $\mathcal{V}_{\text {ext }}$ and apply this construction to the study of intertwining operators and logarithmic modules.

\footnotetext{
${ }^{1}$ We emphasise a term $\frac{c_{L}-2}{24}$ for its importance in a free field realization of $\mathcal{H}$ (see [4] for details).
} 


\section{Lie algebra $W(2,2)$ and the twisted Heisenberg-Virasoro Lie algebra at level zero}

$W(2,2)$ is a Lie algebra with basis $\left\{L(n), W(n), C_{L}, C_{W}: n \in \mathbb{Z}\right\}$ over $\mathbb{C}$, and a Lie bracket

$$
\begin{aligned}
& {[L(n), L(m)]=(n-m) L(n+m)+\delta_{n,-m} \frac{n^{3}-n}{12} C_{L},} \\
& {[L(n), W(m)]=(n-m) W(n+m)+\delta_{n,-m} \frac{n^{3}-n}{12} C_{W},} \\
& {[W(n), W(m)]=\left[\cdot, C_{L}\right]=\left[\cdot, C_{W}\right]=0 .}
\end{aligned}
$$

Highest weight representation theory over $W(2,2)$ was studied in $[14,19]$. However, representations treated in these papers have equal central charges $C_{L}=C_{W}$. These results have recently been generalised to $C_{L} \neq C_{W}$ in [15]. Here we state the most important results. Verma module with central charge $\left(c_{L}, c_{W}\right)$ and highest weight $\left(h, h_{W}\right)$ is denoted by $V^{W(2,2)}\left(c_{L}, c_{W}, h, h_{W}\right)$, its highest weight vector by $v_{h, h_{W}}$ and irreducible quotient module by $L^{W(2,2)}\left(c_{L}, c_{W}, h, h_{W}\right)$.

Recall the definition of a cosingular vector. Homogeneous vector $v \in M$ is called cosingular (or subsingular) if it is not singular in $M$ and if there is a proper submodule $N \subset M$ such that $v+N$ is a singular vector in $M / N$.

Theorem $2.1([15,19])$. Let $c_{W} \neq 0$.

(i) Verma module $V^{W(2,2)}\left(c_{L}, c_{W}, h, h_{W}\right)$ is reducible if and only if $h_{W}=\frac{1-p^{2}}{24} c_{W}$ for some $p \in \mathbb{Z}_{>0}$. In that case, there exists a singular vector $u_{p}^{\prime} \in \mathbb{C}[W(-1), \ldots, W(-p)] v_{h, h_{W}}$ such that $U(W(2,2)) u_{p}^{\prime} \cong V^{W(2,2)}\left(c_{L}, c_{W}, h+p, h_{W}\right)$.

(ii) A quotient module ${ }^{2}$

$$
V^{W(2,2)}\left(c_{L}, c_{W}, h, h_{W}\right) / U(W(2,2)) u_{p}^{\prime}=: \widetilde{L}^{W(2,2)}\left(c_{L}, c_{W}, h_{p, r}, h_{W}\right)
$$

is reducible if and only if $h=h_{p, r}$ for some $r \in \mathbb{Z}_{>0}$. In that case, there is a cosingular vector $u_{r p} \in V^{W(2,2)}\left(c_{L}, c_{W}, h, h_{W}\right)_{h+r p}$ such that $\overline{u_{r p}}:=u_{r p}+U(W(2,2)) u_{p}^{\prime}$ is a singular vector in $\widetilde{L}^{W(2,2)}\left(c_{L}, c_{W}, h_{p, r}, h_{W}\right)$ which generates a submodule isomorphic to $L^{W(2,2)}\left(c_{L}, c_{W}, h_{p, r}+r p, h_{W}\right)$. The short sequence

$$
\begin{aligned}
0 & \rightarrow L^{W(2,2)}\left(c_{L}, c_{W}, h_{p, r}+r p, h_{W}\right) \rightarrow \widetilde{L}^{W(2,2)}\left(c_{L}, c_{W}, h_{p, r}, h_{W}\right) \\
& \rightarrow L^{W(2,2)}\left(c_{L}, c_{W}, h_{p, r}, h_{W}\right) \rightarrow 0
\end{aligned}
$$

where the highest weight vector in $L^{W(2,2)}\left(c_{L}, c_{W}, h_{p, r}+r p, h_{W}\right)$ maps to $\overline{u_{r p}}$ is exact.

Define

$$
\mathcal{A} \mathcal{T}_{W(2,2)}\left(c_{L}, c_{W}\right)=\left\{\left(h_{p, r}, \frac{1-p^{2}}{24} c_{W}\right) \mid p, r \in \mathbb{Z}_{>0}\right\} .
$$

Remark 2.2. We will refer to the (modules of) highest weights $\left(h, h_{W}\right) \in \mathcal{A T}_{W(2,2)}\left(c_{L}, c_{W}\right)$ as atypical for $W(2,2)$, and otherwise as typical. Again, we refer to a highest weight $W(2,2)$ module as (a)typical depending on its highest weight. So a Verma module over $W(2,2)$ contains a nontrivial cosingular vector if and only if it is atypical.

Proposition 2.3. Let $h_{W}=\frac{1-p^{2}}{24} c_{W}, p \in \mathbb{Z}_{>0}$.

\footnotetext{
${ }^{2}$ This module is denoted by $L^{\prime}$ in $[15,19]$. We change notation to $\widetilde{L}$ due to use of superscript $W(2,2)$.
} 
(i) Let $\left(h_{p, r}, h_{W}\right), r \in \mathbb{Z}_{>0}$ be an atypical weight and $k \in \mathbb{Z}$. Then $\left(h_{p, r}+k p, h_{W}\right)$ is atypical if and only if $k<\frac{r}{2}$.

(ii) Atypical Verma module $V^{W(2,2)}\left(h_{p, r}, h_{W}\right)$ contains exactly $\left\lfloor\frac{r+1}{2}\right\rfloor$ cosingular vectors. The weights of these vectors are $h_{p, r}+(r-i) p=h_{p,-r+2 i}, i=0, \ldots,\left\lfloor\frac{r-1}{2}\right\rfloor$.

Proof. (i) Directly from Theorem 2.1 since $h_{p, r}+k p=h_{p, r-2 k}$.

(ii) Follows from (i) since $V^{W(2,2)}\left(h_{p, r}, h_{W}\right)$ contains an infinite chain of submodules isomorphic to Verma modules of highest weights $h_{p, r}+i p=h_{p, r-2 i}, i>0$. Applying Theorem 2.1 to each of these submodules we obtain cosingular vectors of weights

$$
h_{p, r-2 i}+(r-2 i) p=h_{p, r}+(r-i) p=h_{p,-r+2 i}
$$

as long as $r-2 i>0$.

Remark 2.4. Standard PBW basis for $V^{W(2,2)}\left(c_{L}, c_{W}, h, h_{W}\right)$ consists of vectors

$$
W\left(-m_{s}\right) \cdots W\left(-m_{1}\right) L\left(-n_{t}\right) \cdots L\left(-n_{1}\right) v_{h, h_{W}}
$$

such that $m_{s} \geq \cdots \geq m_{1} \geq 1, n_{t} \geq \cdots \geq n_{1} \geq 1$. The only nonzero component of $u_{r p}$ belonging to $\mathbb{C}[L(-1), L(-2), \ldots] v$ is $L(-p)^{r} v_{h, h_{W}}[19]$.

Define $P_{2}(n)=\sum_{i=0}^{n} P(n-i) P(i)$ where $P$ is a partition function with $P(0)=1$. We have the following character formulas [19]

$$
\operatorname{char} V^{W(2,2)}\left(c_{L}, c_{W}, h, h_{W}\right)=q^{h} \sum_{n \geq 0} P_{2}(n) q^{n}=q^{h} \prod_{k \geq 1}\left(1-q^{k}\right)^{-2}
$$

for all $h, h_{W} \in \mathbb{C}$. If $h_{W}=\frac{1-p^{2}}{24} c_{W}$, then

$$
\operatorname{char} \widetilde{L}^{W(2,2)}\left(c_{L}, c_{W}, h, h_{W}\right)=q^{h}\left(1-q^{p}\right) \sum_{n \geq 0} P_{2}(n) q^{n}=q^{h}\left(1-q^{p}\right) \prod_{k \geq 1}\left(1-q^{k}\right)^{-2} .
$$

If $\left(h, h_{W}\right)$ is typical for $W(2,2)$, then this is the character of an irreducible highest weight module. Finally, the character of atypical irreducible module is

$$
\begin{aligned}
\operatorname{char} L^{W(2,2)}\left(c_{L}, c_{W}, h_{p, r}, h_{W}\right) & =q^{h_{p, r}}\left(1-q^{p}\right)\left(1-q^{r p}\right) \sum_{n \geq 0} P_{2}(n) q^{n} \\
& =q^{h_{p, r}}\left(1-q^{p}\right)\left(1-q^{r p}\right) \prod_{k \geq 1}\left(1-q^{k}\right)^{-2} .
\end{aligned}
$$

The twisted Heisenberg-Virasoro algebra $\mathcal{H}$ is the universal central extension of the Lie algebra of differential operators on a circle of order at most one. It is the infinite-dimensional complex Lie algebra with a basis

$$
\{L(n), I(n): n \in \mathbb{Z}\} \cup\left\{C_{L}, C_{L I}, C_{I}\right\}
$$

and commutation relations

$$
\begin{aligned}
& {[L(n), L(m)]=(n-m) L(n+m)+\delta_{n,-m} \frac{n^{3}-n}{12} C_{L},} \\
& {[L(n), I(m)]=-m I(n+m)-\delta_{n,-m}\left(n^{2}+n\right) C_{L I},} \\
& {[I(n), I(m)]=n \delta_{n,-m} C_{I}, \quad\left[\mathcal{H}, C_{L}\right]=\left[\mathcal{H}, C_{L I}\right]=\left[\mathcal{H}, C_{I}\right]=0 .}
\end{aligned}
$$


The Lie algebra $\mathcal{H}$ admits the following triangular decomposition

$$
\begin{aligned}
& \mathcal{H}=\mathcal{H}^{-} \oplus \mathcal{H}^{0} \oplus \mathcal{H}^{+}, \\
& \mathcal{H}^{ \pm}=\operatorname{span}_{\mathbb{C}}\left\{I( \pm n), L( \pm n) \mid n \in \mathbb{Z}_{>0}\right\}, \quad \mathcal{H}^{0}=\operatorname{span}_{\mathbb{C}}\left\{I(0), L(0), C_{L}, C_{L, I}, C_{I}\right\} .
\end{aligned}
$$

Although they seem to be two similar extensions of the Virasoro algebra, representation theories of $W(2,2)$ and $\mathcal{H}$ are different. The main reason for that lies in the fact that $I(0)$ is a central element, while $W(0)$ is not. However, applying free field realization, we shall see that highest weight modules over the two algebras are related.

Denote by $V^{\mathcal{H}}\left(c_{L}, c_{I}, c_{L, I}, h, h_{I}\right)$ the Verma module and by $v_{h, h_{I}}$ its highest weight vector. $C_{L}, C_{I}, C_{L, I}, L(0)$ and $I(0)$ act on $v_{h, h_{I}}$ by scalars $c_{L}, c_{I}, c_{L, I}, h$ and $h_{I}$, respectively. Then $\left(c_{L}, c_{I}, c_{L, I}\right)$ is called a central charge, and $\left(h, h_{I}\right)$ a highest weight. In this paper we consider central charges $\left(c_{L}, 0, c_{L, I}\right)$ such that $c_{L, I} \neq 0$.

Theorem $2.5([8])$. Let $c_{L, I} \neq 0$. Verma module $V^{\mathcal{H}}\left(c_{L}, 0, c_{L, I}, h, h_{I}\right)$ is reducible if and only if $h_{I}=(1 \pm p) c_{L, I}$ for some $p \in \mathbb{Z}_{>0}$. In that case, there is a singular vector $v_{p}^{ \pm}$of weight $p$, which generates a maximal submodule in $V^{\mathcal{H}}\left(c_{L}, 0, c_{L, I}, h, h_{I}\right)$ isomorphic to $V^{\mathcal{H}}\left(c_{L}, 0, c_{L, I}, h+p, h_{I}\right)$.

Remark 2.6. In case $h_{I}=(1+p) c_{L, I}$ an explicit formula for a singular vector $v_{p}^{+}$is obtained using Schur polynomials in $I(-1), \ldots, I(-p)$. See [4] for details. Assume that $x \in U(W(2,2))_{-}$ is such that $x v_{h, h_{I}} \in V^{\mathcal{H}}\left(c_{L}, 0, c_{L, I}, h, h_{I}\right)$ lies in a maximal submodule. Then $x$ does not have a nontrivial additive component (in PBW basis) that belongs to $\mathbb{C}[L(-1), L(-2), \ldots][8]$.

There is an infinite chain of Verma submodules generated by singular vectors $v_{k p}^{ \pm}, k \in \mathbb{Z}_{>0}$, with all the subquotients being irreducible. Note that there is no mention of $\widetilde{L}^{\mathcal{H}}$ since there are no cosingular vectors in $V^{\mathcal{H}}$.

The following character formulas were obtained in [8]:

$$
\begin{aligned}
& \operatorname{char} V^{\mathcal{H}}\left(c_{L}, 0, c_{L}, h, h_{I}\right)=q^{h} \sum_{n \geq 0} P_{2}(n) q^{n}=q^{h} \prod_{k \geq 1}\left(1-q^{k}\right)^{-2}, \\
& \operatorname{char} L^{\mathcal{H}}\left(c_{L}, 0, c_{L}, h, h_{I}\right)=q^{h}\left(1-q^{p}\right) \sum_{n \geq 0} P_{2}(n) q^{n}=q^{h}\left(1-q^{p}\right) \prod_{k \geq 1}\left(1-q^{k}\right)^{-2} .
\end{aligned}
$$

Remark 2.7. Throughout the rest of the paper we work with highest weight modules over the Lie algebras $W(2,2)$ and $\mathcal{H}$ so we always denote algebra in superscript. In order to avoid too cumbersome notation, we omit central charges. Therefore, we write $V^{\mathcal{H}}\left(h, h_{I}\right)$ for Verma module over $\mathcal{H}, V^{W(2,2)}\left(h, h_{W}\right)$ for Verma module over $W(2,2)$ and so on. We always assume that $c_{W}$ and $c_{L, I}$ are nonzero. Moreover, if we work with several modules over both algebras, $c_{L}$ is equal for all modules.

We shall write $\langle x\rangle_{W(2,2)}$ for a cyclic submodule $U(W(2,2)) x$ and $\langle x\rangle_{\mathcal{H}}$ for $U(\mathcal{H}) x$. Finally, $\cong_{W(2,2)}$ denotes an isomorphism of $W(2,2)$-modules.

\section{$3 \quad$ Irreducible highest weight modules}

In this section we present main results of the paper which completely describe the structure of (irreducible) highest weight modules for $\mathcal{H}$ as $W(2,2)$-modules. The main tool is the homomorphism between $W(2,2)$ and the Heisenberg-Virasoro vertex algebras from [4].

$L^{W(2,2)}\left(c_{L}, c_{W}, 0,0\right)$ is a simple universal vertex algebra associated to Lie algebra $W(2,2)$ (cf. $[19,20])$ which we denote by $L^{W(2,2)}\left(c_{L}, c_{W}\right)$. It is generated by fields

$$
L(z)=Y(\omega, z)=\sum_{n \in \mathbb{Z}} L(n) z^{-n-2}, \quad W(z)=Y(W, z)=\sum_{n \in \mathbb{Z}} W(n) z^{-n-2},
$$


where $\omega=L(-2) \mathbf{1}$ and $W=W(-2) \mathbf{1}$. Each highest weight $W(2,2)$-module is also a module over a vertex operator algebra $L^{W(2,2)}\left(c_{L}, c_{W}\right)$.

Likewise (see [7]) $L^{\mathcal{H}}\left(c_{L}, 0, c_{L, I}, 0,0\right)$ is a simple Heisenberg-Virasoro vertex operator algebra, which we denote by $L^{\mathcal{H}}\left(c_{L}, c_{L, I}\right)$. This algebra is generated by the fields

$$
L(z)=Y(\omega, z)=\sum_{n \in \mathbb{Z}} L(n) z^{-n-2}, \quad I(z)=Y(I, z)=\sum_{n \in \mathbb{Z}} I(n) z^{-n-1},
$$

where $\omega=L(-2) \mathbf{1}$ and $I=I(-1) \mathbf{1}$. Moreover, highest weight $\mathcal{H}$-modules are modules over a vertex operator algebra $L^{\mathcal{H}}\left(c_{L}, c_{L, I}\right)$.

It was shown in [4] that there is a monomorphism of vertex operator algebras

$$
\begin{aligned}
\Psi: L^{W(2,2)}\left(c_{L}, c_{W}\right) & \rightarrow L^{\mathcal{H}}\left(c_{L}, c_{L, I}\right), \\
\omega & \mapsto L(-2) \mathbf{1}, \\
W & \mapsto\left(I(-1)^{2}+2 c_{L, I} I(-2)\right) \mathbf{1},
\end{aligned}
$$

where $c_{W}=-24 c_{L, I}^{2}$. By means of $\Psi$, each highest weight module over $\mathcal{H}$ becomes an $L^{W(2,2)}\left(c_{L}, c_{W}\right)$-module and therefore a module over $W(2,2)$. In particular, $\Psi$ induces a nontrivial $W(2,2)$-homomorphism (which we shall denote by the same letter)

$$
\Psi: V^{W(2,2)}\left(c_{L}, c_{W}, h, h_{W}\right) \rightarrow V^{\mathcal{H}}\left(c_{L}, 0, c_{L, I}, h, h_{I}\right),
$$

where $c_{W}=-24 c_{L, I}^{2}$ and $h_{W}=h_{I}\left(h_{I}-2 c_{L, I}\right) . \Psi$ maps the highest weight vector $v_{h, h_{W}}$ to the highest weight vector $v_{h, h_{I}}$ and the action of $W(-n)$ on $V^{\mathcal{H}}\left(c_{L}, 0, c_{L, I}, h, h_{I}\right)$ is given by

$$
\begin{aligned}
& W(-n) \equiv 2 c_{L, I}(n-1) I(-n)+\sum_{i \in \mathbb{Z}} I(-i) I(-n+i), \\
& W(-n) \equiv 2 c_{L, I}\left(n-1+\frac{h_{I}}{c_{L, I}}\right) I(-n)+\sum_{i \neq 0, n} I(-i) I(-n+i) .
\end{aligned}
$$

Note that $h_{W}=\frac{1-p^{2}}{24} c_{W}$ if and only if $h_{I}=(1 \pm p) c_{L, I}$, so either both of these Verma modules are irreducible, or they are reducible with singular vectors at equal levels. Moreover, $\left(h, h_{W}\right) \in \mathcal{A T}_{W(2,2)}\left(c_{L}, c_{W}\right)$ if and only if $\left(h, h_{I}\right) \in \mathcal{A} \mathcal{T}_{\mathcal{H}}\left(c_{L}, c_{L, I}\right)$.

Throughout the rest of this section we assume that $c_{W}=-24 c_{L, I}^{2}$.

Lemma 3.1 ([4, Lemma 7.2]). Suppose that $h_{I} \neq(1-p) c_{L, I}$ for all $p \in \mathbb{Z}_{>0}$. Then $\Psi$ is an isomorphism of $W(2,2)$-modules. In particular, if $h_{I} \neq(1 \pm p) c_{L, I}$ for $p \in \mathbb{Z}_{>0}$, then

$$
L^{\mathcal{H}}\left(h, h_{I}\right) \cong_{W(2,2)} L^{W(2,2)}\left(h, h_{W}\right),
$$

where $h_{W}=h_{I}\left(h_{I}-2 c_{L, I}\right)$.

Lemma 3.2. Suppose that $x \in V^{\mathcal{H}}\left(h, h_{I}\right)$ is $\mathcal{H}$-singular. Then $x$ is $W(2,2)$-singular as well. In particular, if $y$ is a homogeneous vector such that $x=\Psi(y)$, then $y$ is either singular or cosingular vector in $V^{W(2,2)}\left(h, h_{W}\right)$.

Proof. Let $x \in V^{\mathcal{H}}\left(h, h_{I}\right)$ be a $\mathcal{H}$-singular vector, i.e., $L(k) x=I(k) x=0$ for all $k \in \mathbb{Z}_{>0}$. From (3.2) we have

$$
W(n) x=-2 c_{L, I}(n+1) I(n) x+\sum_{i \in \mathbb{Z}} I(-i) I(n+i) x,
$$

so $W(n) x=0$ for all $n \in \mathbb{Z}_{>0}$. Therefore, $x$ is $W(2,2)$-singular. If $x=\Psi(y)$, then $L(k) y, W(k) y$ $\in \operatorname{Ker} \Psi$ for $k>0$. Therefore $y+\operatorname{Ker} \Psi$ is a singular vector in $V^{W(2,2)}\left(h, h_{W}\right) / \operatorname{Ker} \Psi$. 
Theorem 3.3. Let $p \in \mathbb{Z}_{>0}$.

(i) If $\left(h,(1+p) c_{L, I}\right)$ is typical for $\mathcal{H}$ (equivalently if $\left(h, \frac{1-p^{2}}{24} c_{W}\right)$ is typical for $\left.W(2,2)\right)$ then

$$
L^{\mathcal{H}}\left(h,(1+p) c_{L, I}\right) \cong_{W(2,2)} L^{W(2,2)}\left(h, \frac{1-p^{2}}{24} c_{W}\right) .
$$

(ii) If $\left(h_{p, r},(1+p) c_{L, I}\right) \in \mathcal{A} \mathcal{T}_{\mathcal{H}}\left(c_{L}, c_{L, I}\right)$ (equivalently if $\left.\left(h_{p, r}, \frac{1-p^{2}}{24} c_{W}\right) \in \mathcal{A T}_{W(2,2)}\left(c_{L}, c_{W}\right)\right)$ then

$$
L^{\mathcal{H}}\left(h_{p, r},(1+p) c_{L, I}\right) \cong_{W(2,2)} \widetilde{L}^{W(2,2)}\left(h_{p, r}, \frac{1-p^{2}}{24} c_{W}\right)
$$

and the short sequence of $W(2,2)$-modules

$$
\begin{aligned}
0 & \rightarrow L^{W(2,2)}\left(h_{p, r}+r p, \frac{1-p^{2}}{24} c_{W}\right) \rightarrow L^{\mathcal{H}}\left(h_{p, r},(1+p) c_{L, I}\right) \\
& \rightarrow L^{W(2,2)}\left(h_{p, r}, \frac{1-p^{2}}{24} c_{W}\right) \rightarrow 0
\end{aligned}
$$

is exact.

Proof. By Lemma 3.1, $\Psi$ is an isomorphism of Verma modules and thus by Lemma 3.2 it maps a $W(2,2)$-singular vector $u_{p}^{\prime}$ to an $\mathcal{H}$-singular vector $v_{p}^{+}$. If $h \neq h_{p, r}$, both of these vectors generate maximal submodules in respective Verma modules so (3.3) follows.

Now suppose that $h=h_{p, r}$. We need to show that a cosingular vector $u_{r p}$ is not mapped into a maximal submodule of $V^{\mathcal{H}}\left(h_{p, r}, h_{I}\right)$. But $u_{r p}$ has $L(-p)^{r} v$ as an additive component (see Remark 2.4), and by construction (3.1), $\Psi\left(u_{r p}\right)$ also must have this additive component. However, $\Psi\left(u_{r p}\right)$ can not lie in a maximal $\mathcal{H}$-submodule of $V^{\mathcal{H}}\left(h, h_{I}\right)$ (see Remark 2.6). This means that isomorphism $\Psi$ of Verma modules induces a $W(2,2)$-isomorphism of $\widetilde{L}^{W(2,2)}\left(h, h_{W}\right)$ and $L^{\mathcal{H}}\left(h, h_{I}\right)$ for all $h \in \mathbb{C}$. Exactness of (3.4) is just an application of (2.1).

Remark 3.4. Note that the image $\Psi\left(u_{r p}\right)$ of a $W(2,2)$-cosingular vector is neither $\mathcal{H}$-singular, nor $\mathcal{H}$-cosingular in $V^{\mathcal{H}}\left(h_{p, r},(1+p) c_{L, I}\right)$. For example, $L(-1) v_{0,0}$ in $V^{\mathcal{H}}\left(0,2 c_{L, I}\right)$ is $W(2,2)$ cosingular, but not $\mathcal{H}$-singular since $I(1) L(-1) v_{0,0}=2 c_{L, I} v_{0,0}$.

If $h_{I}=(1-p) c_{L, I}$, then $\Psi$ is not an isomorphism. We shall present a $W(2,2)$-structure of Verma module later. In order to examine irreducible $W(2,2)$-modules we apply the properties of contragredient modules.

Let us recall the definition of contragredient module (see [12]). Assume that $\left(M, Y_{M}\right)$ is a graded module over a vertex operator algebra $V$ such that $M=\oplus_{n=0}^{\infty} M(n), \operatorname{dim} M(n)<\infty$ and suppose that there is $\gamma \in \mathbb{C}$ such that $L(0) \mid M(n) \equiv(\gamma+n)$ Id. The contragredient module $\left(M^{*}, Y_{M^{*}}\right)$ is defined as follows. For every $n \in \mathbb{Z}_{>0}$ let $M(n)^{*}$ be the dual vector space and let $M^{*}=\oplus_{n=0}^{\infty} M(n)^{*}$ be a restricted dual of $M$. Consider the natural pairing $\langle\cdot, \cdot\rangle: M^{*} \otimes M \rightarrow \mathbb{C}$. Define the linear map $Y_{M^{*}}: V \rightarrow$ End $M^{*}\left[\left[z, z^{-1}\right]\right]$ such that

$$
\left\langle Y_{M^{*}}(v, z) m^{\prime}, m\right\rangle=\left\langle m^{\prime}, Y_{M}\left(e^{z L(1)}\left(-z^{-2}\right)^{L(0)} v, z^{-1}\right) m\right\rangle
$$

for each $v \in V, m \in M, m^{\prime} \in M^{*}$. Then $\left(M^{*}, Y_{M^{*}}\right)$ is a $V$-module.

In particular, choosing $v=\omega=L_{-2} \mathbf{1}$ in (3.5) one gets

$$
\left\langle L(n) m^{\prime}, m\right\rangle=\left\langle m^{\prime}, L(-n) m\right\rangle .
$$

Simple calculation with $I \in L^{\mathcal{H}}\left(c_{L}, c_{L, I}\right)$ and $W \in L^{W(2,2)}\left(c_{L}, c_{W}\right)$ shows that

$$
\left\langle I(n) m^{\prime}, m\right\rangle=\left\langle m^{\prime},-I(-n) m+\delta_{n, 0} 2 c_{L, I}\right\rangle, \quad\left\langle W(n) m^{\prime}, m\right\rangle=\left\langle m^{\prime}, W(-n) m\right\rangle .
$$

Therefore we get the following result (the first and third relations were given in [4]): 


\section{Lemma 3.5.}

$$
L^{\mathcal{H}}\left(h, h_{I}\right)^{*} \cong L^{\mathcal{H}}\left(h,-h_{I}+2 c_{L, I}\right), \quad L^{W(2,2)}\left(h, h_{W}\right)^{*} \cong L^{W(2,2)}\left(h, h_{W}\right) .
$$

In particular,

$$
L^{\mathcal{H}}\left(h,(1 \pm p) c_{L, I}\right)^{*} \cong L^{\mathcal{H}}\left(h,(1 \mp p) c_{L, I}\right) .
$$

Directly from Theorem 3.3 and Lemma 3.5 follows

Corollary 3.6. Let $p \in \mathbb{Z}_{>0}$.

(i) If $\left(h,(1-p) c_{L, I}\right)$ is typical for $\mathcal{H}$ (equivalently if $\left(h, \frac{1-p^{2}}{24} c_{W}\right)$ is typical for $\left.W(2,2)\right)$ then

$$
L^{\mathcal{H}}\left(h,(1-p) c_{L, I}\right) \cong_{W(2,2)} L^{W(2,2)}\left(h, \frac{1-p^{2}}{24} c_{W}\right) .
$$

(ii) If $\left(h_{p, r},(1-p) c_{L, I}\right) \in \mathcal{A} \mathcal{T}_{\mathcal{H}}\left(c_{L}, c_{L, I}\right)$ (equivalently if $\left.\left(h_{p, r}, \frac{1-p^{2}}{24} c_{W}\right) \in \mathcal{A T}_{W(2,2)}\left(c_{L}, c_{W}\right)\right)$ then

$$
L^{\mathcal{H}}\left(h_{p, r},(1-p) c_{L, I}\right) \cong_{W(2,2)} \widetilde{L}^{W(2,2)}\left(h_{p, r}, \frac{1-p^{2}}{24} c_{W}\right)^{*}
$$

and the short sequence of $W(2,2)$-modules

$$
\begin{aligned}
0 & \rightarrow L^{W(2,2)}\left(h_{p, r}, \frac{1-p^{2}}{24} c_{W}\right) \rightarrow L^{\mathcal{H}}\left(h_{p, r},(1-p) c_{L, I}\right) \\
& \rightarrow L^{W(2,2)}\left(h_{p, r}+r p, \frac{1-p^{2}}{24} c_{W}\right) \rightarrow 0
\end{aligned}
$$

is exact.

From Lemma 3.1, Theorem 3.3 and Corollary 3.6 follow assertions of Theorem 1.1.

Finally, we show that Verma module over $\mathcal{H}$ is an infinite direct sum of irreducible $W(2,2)$ modules. Recall that $V^{\mathcal{H}}\left(h,(1-p) c_{L, I}\right)$ has a series of singular vectors $v_{i p}^{-}, i \in \mathbb{Z}_{\geq 0}$ (for $i=0$, we set $\left.v_{0}^{-}=v_{h, h_{I}}\right)$ which generate a descending chain of Verma submodules over $\mathcal{H}$ :

$$
\begin{gathered}
\left\langle v_{h, h_{I}}\right\rangle_{\mathcal{H}}=V^{\mathcal{H}}\left(h, h_{I}\right) \\
\cup I \\
\left\langle v_{p}^{-}\right\rangle_{\mathcal{H}} \cong V^{\mathcal{H}}\left(h+p, h_{I}\right) \\
\cup I \\
\vdots \\
\left\langle v_{i p}^{-}\right\rangle_{\mathcal{H}} \cong V^{\mathcal{H}}\left(h+i p, h_{I}\right) \\
\left.\cup v_{(i+1) p}^{-}\right\rangle_{\mathcal{H}} \cong V^{\mathcal{H}}\left(h+(i+1) p, h_{I}\right) \\
\cup I \\
\vdots
\end{gathered}
$$

Therefore one may identify $V^{\mathcal{H}}\left(h+i p, h_{I}\right)$ with a submodule of $V^{\mathcal{H}}\left(h, h_{I}\right)$ and a singular vector $v_{i p}^{-} \in V^{\mathcal{H}}\left(h, h_{I}\right)$ with the highest weight vector $v_{h+i p, h_{I}} \in V^{\mathcal{H}}\left(h+i p, h_{I}\right)$. We will prove that in a typical case each of those vectors generates an irreducible $W(2,2)$-submodule. 
Theorem 3.7. Let $p \in \mathbb{Z}_{>0}$. Suppose that $\left(h,(1-p) c_{L, I}\right) \notin \mathcal{A} \mathcal{T}_{\mathcal{H}}\left(c_{L}, c_{L, I}\right)$. Then we have the following isomorphism of $W(2,2)$-modules

$$
V^{\mathcal{H}}\left(h,(1-p) c_{L, I}\right) \cong_{W(2,2)} \bigoplus_{i \geq 0} L^{W(2,2)}\left(h+i p, \frac{1-p^{2}}{24} c_{W}\right)
$$

Proof. First we notice that the vertex algebra homorphism $\Psi: L^{W(2,2)}\left(c_{W}, c_{L}\right) \rightarrow L^{\mathcal{H}}\left(c_{W}, c_{L}\right)$, for every $i \in \mathbb{Z}_{\geq 0}$ induces the following non-trivial homomorphism of $W(2,2)$-modules:

$$
\Psi^{(i)}: V^{W(2,2)}\left(h+i p, \frac{1-p^{2}}{24} c_{W}\right) \rightarrow\left\langle v_{i p}^{-}\right\rangle_{W(2,2)} \subset V^{\mathcal{H}}\left(h+i p,(1-p) c_{L, I}\right),
$$

which maps the highest weight vector of $V^{W(2,2)}\left(h+i p, \frac{1-p^{2}}{24} c_{W}\right)$ to $v_{i p}^{-}$. Since $\left(h, \frac{1-p^{2}}{24} c_{W}\right)$ is typical it follows from Proposition 2.3(i) that $\left(h+i p, \frac{1-p^{2}}{24} c_{W}\right)$ are typical for all $i \in \mathbb{Z}_{>0}$ as well.

Let $h_{W}=\frac{1-p^{2}}{24} c_{W}$. Consider the homomorphism $\Psi^{(i)}: V^{W(2,2)}\left(h+i p, h_{W}\right) \rightarrow V^{\mathcal{H}}\left(h+i p, h_{I}\right)$ above. Applying (3.2), we get

$$
\Psi^{(i)}\left(W(-p) v_{h+i p, h_{W}}\right)=\sum_{i=1}^{p-1} I(-i) I(i-p) v_{h+i p, h_{I}},
$$

so $I(-p) v_{h+i p, h_{I}} \notin \operatorname{Im} \Psi^{(i)}$. Since the Verma modules $V^{W(2,2)}\left(h+i p, h_{W}\right)$ and $V^{\mathcal{H}}\left(h+i p, h_{I}\right)$ have equal characters, it follows that $\operatorname{Ker} \Psi^{(i)}$ contains a singular vector in $V^{W(2,2)}\left(h+i p, h_{W}\right)$ of conformal weight $h+(i+1) p$. Since the weight $\left(h+i p, h_{W}\right)$ is typical, the maximal submodule in $V^{W(2,2)}\left(h+i p, h_{W}\right)$ is generated by this singular vector so we conclude that $\operatorname{Ker} \Psi^{(i)}$ is the maximal submodule in $V^{W(2,2)}\left(h+i p, h_{W}\right)$. Therefore

$$
\operatorname{Im} \Psi^{(i)}=\left\langle v_{h+i p, h_{I}}\right\rangle_{W(2,2)} \cong L^{W(2,2)}\left(h+i p, h_{W}\right) .
$$

In this way we get a series of $W(2,2)$-monomorphisms

$$
L^{W(2,2)}\left(h+i p, h_{W}\right) \hookrightarrow V^{\mathcal{H}}\left(h,(1-p) c_{L, I}\right), \quad i \in \mathbb{Z}_{\geq 0}
$$

mapping $v_{h+i p, h_{W}}$ to a singular vector $v_{i p}^{-}$. Let $v_{j p}^{-}$be an $\mathcal{H}$-singular vector in $V^{\mathcal{H}}(h+i p,(1-$ $\left.p) c_{L, I}\right)$ of weight $h+j p$, for $j>i$. By Lemma 3.2, $v_{j p}^{-}$is singular for $W(2,2)$ and therefore $v_{j p}^{-} \notin\left\langle v_{h+i p, h_{I}}\right\rangle_{W(2,2)}$ for $j>i$. We conclude that the images of morphisms (3.6) have trivial pairwise intersections (since these images are non-isomorphic irreducible modules), so their sum is direct. The assertion follows from the observation that the character of this sum is

$$
\sum_{i=0}^{\infty} q^{h+i p}\left(1-q^{p}\right) \prod_{k \geq 1}\left(1-q^{k}\right)^{-2}=q^{h} \prod_{k \geq 1}\left(1-q^{k}\right)^{-2}=\operatorname{char} V^{\mathcal{H}}\left(h,(1-p) c_{L . I}\right) .
$$

Remark 3.8. It is interesting to notice that our Theorem 3.7 shows that $V^{\mathcal{H}}\left(h, h_{I}\right)$ can be considered as a $W(2,2)$-analogue of certain Feigin-Fuchs modules for the Virasoro algebra which are also direct sums of infinitely many irreducible modules (cf. [11], [2, Theorem 5.1]).

From the previous theorem follows

$$
\begin{gathered}
V^{\mathcal{H}}\left(h, h_{I}\right) \\
\| \\
\left\langle v_{h, h_{I}}\right\rangle_{W(2,2)}=L^{W(2,2)}\left(h, h_{W}\right) \\
\oplus
\end{gathered}
$$




$$
\begin{gathered}
\left\langle v_{p}^{-}\right\rangle_{W(2,2)} \cong L^{W(2,2)}\left(h+p, h_{W}\right) \\
\oplus \\
\vdots \\
\oplus \\
\left\langle v_{i p}^{-}\right\rangle_{W(2,2)} \cong L^{W(2,2)}\left(h+i p, h_{W}\right)
\end{gathered}
$$

$\oplus$

In atypical case however, the $W(2,2)$-submodules generated by $\mathcal{H}$-singular vectors are nested as follows. Consider $V^{\mathcal{H}}\left(h_{p, r}, h_{I}\right)$ where $\left(h_{p, r}, h_{I}\right) \in \mathcal{A T}_{\mathcal{H}}\left(c_{L}, c_{L, I}\right)$. Then $\Psi^{0}$ maps a cosingular vector $u_{r p} \in V^{W(2,2)}\left(h_{p, r}, h_{W}\right)$ to a singular vector $v_{r p}^{-}$. In other words we have

$$
\left\langle v_{r p}^{-}\right\rangle_{W(2,2)} \subseteq\left\langle v_{h_{p, r}, h_{I}}\right\rangle \cong_{W(2,2)} \widetilde{L}^{W(2,2)}\left(h_{p, r}, h_{W}\right) .
$$

Using the same argument in view of Proposition 2.3 we see that

$$
\left\langle v_{(r-i) p}^{-}\right\rangle_{W(2,2)} \subseteq\left\langle v_{i p}^{-}\right\rangle_{W(2,2)} \cong \widetilde{L}^{W(2,2)}\left(h_{p, r}+i p, h_{W}\right), \quad i=1, \ldots,\left\lfloor\frac{r-1}{2}\right\rfloor .
$$

Therefore,

$$
\begin{array}{ll}
\left\langle v_{h_{p, r}, h_{I}}\right\rangle_{W(2,2)} /\left\langle v_{r p}^{-}\right\rangle_{W(2,2)} \cong L^{W(2,2)}\left(h_{p, r}, h_{W}\right), & \\
\left\langle v_{i p}^{-}\right\rangle_{W(2,2)} /\left\langle v_{(r-i) p}^{-}\right\rangle_{W(2,2)} \cong L^{W(2,2)}\left(h_{p, r}+i p, h_{W}\right), & i<\frac{r-1}{2}, \\
\left\langle v_{i p}^{-}\right\rangle_{W(2,2)} \cong L^{W(2,2)}\left(h_{p, r}+i p, h_{W}\right), & i \geq \frac{r-1}{2} .
\end{array}
$$

In this case, $I(-p)^{r-i} v_{h_{p, r}, h_{I}}$ are $W(2,2)$-cosingular vectors in $V^{\mathcal{H}}\left(h_{p, r}, h_{I}\right)$.

Example 3.9. Consider $p=1$ case. Singular vector in $V^{\mathcal{H}}(h, 0)$ is $u_{1}^{\prime}=\left(L(-1)+\frac{h}{c_{L, I}} I(-1)\right) v_{h, 0}$, and $u_{1}^{\prime}$ generates a copy of $V^{\mathcal{H}}(h+1,0)$.

$r=1: \Psi: V^{W(2,2)}(0,0) \rightarrow V^{\mathcal{H}}(0,0)$ maps a singular vector $u_{1}^{\prime}=W(-1) v_{0,0}$ to 0 and a cosingular vector $u_{1}=L(-1) v_{0,0}$ to $\mathcal{H}$-singular vector $v_{1}^{-}=L(-1) v_{0,0}$. We get the short exact sequence of $W(2,2)$-modules

$$
0 \rightarrow L^{W(2,2)}(0,0) \rightarrow L^{\mathcal{H}}(0,0) \rightarrow L^{W(2,2)}(1,0) \rightarrow 0,
$$

which is an expansion of (3.1) considered as a homomorphism of $W(2,2)$-modules. The rightmost module is generated by a projective image of $I(-1) v_{0,0}$. Therefore, $L^{\mathcal{H}}\left(c_{L}, c_{L, I}\right)$ is generated over $W(2,2)$ by $v_{0,0}$ and $I(-1) v_{0,0}$.

$r \in \mathbb{Z}_{>0}$ : In general, a cosingular vector $u_{r p} \in V^{W(2,2)}\left(\frac{1-r}{2}, 0\right)$ maps to a singular vector $v_{r}^{-} \in V^{\mathcal{H}}\left(\frac{1-r}{2}, 0\right)$ of weight $\frac{1+r}{2}$.

$$
v_{r}^{-}=\prod_{i=0}^{r-1}\left(L(-1)+\frac{1-r+2 i}{2 c_{L, I}} I(-1)\right) v_{\frac{1-r}{2}, 0} .
$$

\section{Screening operators and $W(2,2)$-algebra}

We think that the vertex algebra $L^{W(2,2)}\left(c_{L}, c_{W}\right)$ is a very interesting example of non-rational vertex algebra, which admits similar fusion ring of representations as some $\mathcal{W}$-algebras appearing in LCFT (cf. $[1,2,10,13]$ ). Since $\mathcal{W}$-algebras appearing in LCFT are realized as kernels of 
screening operators acting on certain modules for Heisenberg vertex algebras, it is natural to ask if $L^{W(2,2)}\left(c_{L}, c_{W}\right)$ admits similar realization. In [4] we embedded the $W(2,2)$-algebra as a subalgebra of the Heisenberg-Virasoro vertex algebra. In this section we shall construct a screening operator $S_{1}$ such that the kernel of this operator is exactly $L^{W(2,2)}\left(c_{L}, c_{W}\right)$.

Let us first construct a non-semisimple extension of the vertex algebra $L^{\mathcal{H}}\left(c_{L}, c_{L, I}\right)$. Recall that the Lie algebra $\mathcal{H}$ admits the triangular decomposition (2.2). Let $E=\operatorname{span}_{\mathbb{C}}\left\{v^{0}, v^{1}\right\}$ be 2-dimensional $\mathcal{H}^{\geq 0}=\mathcal{H}^{0} \oplus \mathcal{H}^{+}$-module such that $\mathcal{H}^{+}$acts trivially on $E$ and

$$
\begin{aligned}
& L(0) v^{i}=v^{i}, \quad i=0,1, \quad I(0) v^{1}=v^{0}, \quad I(0) v^{0}=0, \\
& C_{L} v^{i}=c_{L} v^{i}, \quad C_{L, I} v^{i}=c_{L, I} v^{i}, \quad C_{I} v^{i}=0, \quad i=1,2 .
\end{aligned}
$$

Consider now induced $\mathcal{H}$-module

$$
\widetilde{E}=U(\mathcal{H}) \otimes_{U(\mathcal{H} \geq 0)} E .
$$

By construction, $\widetilde{E}$ is a non-split self-extension of the Verma module $V^{\mathcal{H}}(1,0)$ :

$$
0 \rightarrow V^{\mathcal{H}}(1,0) \rightarrow \widetilde{E} \rightarrow V^{\mathcal{H}}(1,0) \rightarrow 0 \text {. }
$$

Moreover, $\widetilde{E}$ is a restricted module for $\mathcal{H}$ and therefore it is a module over vertex operator algebra $L^{\mathcal{H}}\left(c_{L}, c_{L, I}\right)$. Since

$$
\widetilde{E} \cong E \otimes U\left(\mathcal{H}^{-}\right)
$$

as a vector space, the operator $L(0)$ defines a $\mathbb{Z}_{\geq 0}$-gradation on $\widetilde{E}$.

Note that $\left(L(-1)+I(-1) / c_{L, I}\right) v_{0}$ is a singular vector in $\widetilde{E}$ and it generates the proper submodule. Finally we define the quotient module

$$
\mathcal{U}=\frac{\widetilde{E}}{U(\mathcal{H}) \cdot\left(L(-1)+I(-1) / c_{L, I}\right) v_{0}} .
$$

Proposition 4.1. $\mathcal{U}$ is a $\mathbb{Z}_{\geq 0}$-graded module for the vertex operator algebra $L^{\mathcal{H}}\left(c_{L}, c_{L, I}\right)$ :

$$
\mathcal{U}=\bigoplus_{m \in \mathbb{Z} \geq 0} \mathcal{U}(m), \quad L(0) \mid \mathcal{U}(m) \equiv(m+1) \operatorname{Id} .
$$

The lowest component $\mathcal{U}(0) \cong E$. Moreover, $\mathcal{U}$ is a non-split extension of the Verma module $V^{\mathcal{H}}(1,0)$ by the simple highest weight module $L^{\mathcal{H}}(1,0)$ :

$$
0 \rightarrow L^{\mathcal{H}}(1,0) \rightarrow \mathcal{U} \rightarrow V^{\mathcal{H}}(1,0) \rightarrow 0 .
$$

Proof. By construction $\mathcal{U}$ is a graded quotient of a $\mathbb{Z}_{\geq 0}$-graded $L^{\mathcal{H}}\left(c_{L}, c_{L, I}\right)$-module $\widetilde{E}$. The lowest component is $\mathcal{U}(0) \cong E$. Submodule $U(\mathcal{H}) \cdot v^{0}$ is isomorphic to $L^{\mathcal{H}}(1,0)$, and the projective image of $v^{1}$ generates the Verma module $V^{\mathcal{H}}(1,0)$ since $I(0) v^{1}=v^{0}$. For the same reason, this exact sequence does not split.

Now we consider $L^{\mathcal{H}}\left(c_{L}, c_{L, I}\right)$-module

$$
\mathcal{V}^{\mathrm{ext}}:=L^{\mathcal{H}}\left(c_{L}, c_{L, I}\right) \oplus \mathcal{U}
$$

By using [16, Theorem 4.8.1] (see also $[3,17]$ ) we have that $\mathcal{V}^{\text {ext }}$ has the structure of a vertex operator algebra with vertex operator map $Y_{\text {ext }}$ defined as follows:

$$
Y_{\text {ext }}\left(a_{1}+w_{1}, z\right)\left(a_{2}+w_{2}\right)=Y\left(a_{1}, z\right)\left(a_{2}+w_{2}\right)+e^{z L(-1)} Y\left(a_{2},-z\right) w_{1},
$$

where $a_{1}, a_{2} \in L^{\mathcal{H}}\left(c_{L}, c_{L, I}\right), w_{1}, w_{2} \in \mathcal{U}$. 
Take now $v^{i} \in E \subset \mathcal{U}, i=0,1$ as above and define

$$
S_{i}(z)=Y_{\text {ext }}\left(v^{i}, z\right)=\sum_{n \in \mathbb{Z}} S_{i}(n) z^{-n-1} .
$$

By construction

$$
S_{1}(z) \in \text { End }\left(L^{\mathcal{H}}\left(c_{L}, c_{L, I}\right), L^{\mathcal{H}}(1,0)\right)((z)) .
$$

Proposition 4.2. For all $n, m \in \mathbb{Z}$ we have:

$$
\begin{aligned}
& {\left[L(n), S_{i}(m)\right]=-m S_{i}(n+m), \quad i=0,1,} \\
& {\left[W(n), S_{0}(m)\right]=0, \quad\left[W(n), S_{1}(m)\right]=2 m c_{L, I} S_{0}(n+m) .}
\end{aligned}
$$

In particular, $S_{0}(0)$ and $S_{1}(0)$ are screening operators. Moreover,

$$
S_{1}=S_{1}(0): L^{\mathcal{H}}\left(c_{L}, c_{L, I}\right) \rightarrow L^{\mathcal{H}}(1,0)
$$

is nontrivial and $S_{1}(0) I(-1) \mathbf{1}=-v_{0}$.

Proof. Since $L(k) v^{i}=\delta_{k, 0} v^{i}$ for $k \geq 0$, commutator formula gives that

$$
\left[L(n), S_{i}(m)\right]=-m S_{i}(n+m) .
$$

Next we calculate $\left[W(n), S_{1}(m)\right]$. We have

$$
\begin{aligned}
& W(-1) v^{1}=2 I(-1) v^{0}=-2 c_{L, I} L(-1) v^{0}, \\
& W(0) v^{1}=-2 c_{L, I} v^{0}, \quad W(n) v^{1}=0, \quad n \geq 0 .
\end{aligned}
$$

This implies that

$$
\left[W(n), S_{1}(m)\right]=2 c_{L, I} m S_{0}(n+m) .
$$

Since $W(n) v^{0}=0$ for $n \geq-1$ we get

$$
\left[W(n), S_{0}(m)\right]=0 .
$$

Therefore we have proved that $S_{i}(0), i=0,1$ are screening operators. Next we have

$$
S_{1}(0) I(-1) \mathbf{1}=\operatorname{Res}_{z} Y_{\text {ext }}\left(v^{1}, z\right) I(-1)=\operatorname{Res}_{z} e^{z L(-1)} Y(I(-1) \mathbf{1},-z) v^{1}=-v_{0} .
$$

The proof follows.

Theorem 4.3. $S_{1}$ is a derivation of the vertex algebra $\mathcal{V}^{\text {ext }}$ and we have

$$
\operatorname{Ker}_{L^{\mathcal{H}}\left(c_{L}, c_{L, I}\right)} S_{1} \cong L^{W(2,2)}\left(c_{L}, c_{W}\right) .
$$

Proof. By construction $S_{1}=\operatorname{Res}_{z} Y_{\text {ext }}\left(v^{1}, z\right)$, so $S_{1}$ is a derivation so $\bar{W}=\operatorname{Ker}_{L^{\mathcal{H}}\left(c_{L}, c_{L, I}\right)} S_{1}$ is a vertex subalgebra of $L^{\mathcal{H}}\left(c_{L}, c_{L, I}\right)$. Since

$$
S_{1} L(-2) \mathbf{1}=S_{1} W(-2) \mathbf{1}=0
$$

we have that $L^{W(2,2)}\left(c_{L}, c_{W}\right) \subset \bar{W}$. Since $S_{1} I(-1) \mathbf{1} \neq 0$, we have that $I(-1) \mathbf{1}$ does not belong to $\bar{W}$. By using the fact that $L^{\mathcal{H}}\left(c_{L}, c_{L, I}\right)$ is as $W(2,2)$-module generated by singular vector $\mathbf{1}$ and cosingular vector $I(-1) \mathbf{1}$ (see Example 3.9) we get that $\bar{W}=L^{W(2,2)}\left(c_{L}, c_{W}\right)$. The proof follows.

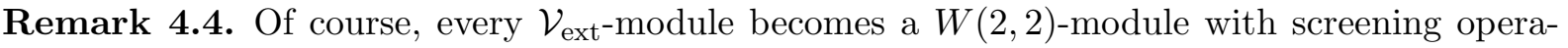
tor $S_{1}$. Similar statement holds for intertwining operators. Constructions of such modules and intertwining operators require different techniques which we will present in our forthcoming paper [5]. 


\section{Acknowledgements}

The authors are partially supported by the Croatian Science Foundation under the project 2634 and by the Croatian Scientific Centre of Excellence QuantixLie.

\section{References}

[1] Adamović D., Classification of irreducible modules of certain subalgebras of free boson vertex algebra, J. Algebra 270 (2003), 115-132, math.QA/0207155.

[2] Adamović D., Milas A., Logarithmic intertwining operators and $W(2,2 p-1)$ algebras, J. Math. Phys. 48 (2007), 073503, 20 pages, math.QA/0702081.

[3] Adamović D., Milas A., Lattice construction of logarithmic modules for certain vertex algebras, Selecta Math. (N.S.) 15 (2009), 535-561, arXiv:0902.3417.

[4] Adamović D., Radobolja G., Free field realization of the twisted Heisenberg-Virasoro algebra at level zero and its applications, J. Pure Appl. Algebra 219 (2015), 4322-4342, arXiv:1405.1707.

[5] Adamović D., Radobolja G., Self-dual and logarithmic representations of the twisted Heisenberg-Virasoro algebra at level zero, in preparation.

[6] Arbarello E., De Concini C., Kac V.G., Procesi C., Moduli spaces of curves and representation theory, Comm. Math. Phys. 117 (1988), 1-36.

[7] Billig Y., Energy-momentum tensor for the toroidal Lie algebra, math.RT/0201313.

[8] Billig Y., Representations of the twisted Heisenberg-Virasoro algebra at level zero, Canad. Math. Bull. 46 (2003), 529-537, math.RT/0201314.

[9] Billig Y., A category of modules for the full toroidal Lie algebra, Int. Math. Res. Not. 2006 (2006), 68395, 46 pages, math.RT/0509368.

[10] Creutzig T., Milas A., False theta functions and the Verlinde formula, Adv. Math. 262 (2014), 520-545, arXiv:1309.6037.

[11] Feigin B.L., Fuchs D.B., Representations of the Virasoro algebra, in Representation of Lie Groups and Related Topics, Adv. Stud. Contemp. Math., Vol. 7, Gordon and Breach, New York, 1990, 465-554.

[12] Frenkel I.B., Huang Y.-Z., Lepowsky J., On axiomatic approaches to vertex operator algebras and modules, Mem. Amer. Math. Soc. 104 (1993), viii+64 pages.

[13] Gainutdinov A.M., Semikhatov A.M., Tipunin I.Yu., Feigin B.L., The Kazhdan-Lusztig correspondence for the representation category of the triplet $W$-algebra in logorithmic conformal field theories, Theoret. Math. Phys. 148 (2006), 1210-1235, math.QA/0512621.

[14] Jiang W., Pei Y., On the structure of Verma modules over the $W$-algebra $W(2,2)$, J. Math. Phys. 51 (2010), 022303, 8 pages.

[15] Jiang W., Zhang W., Verma modules over the W(2,2) algebras, J. Geom. Phys. 98 (2015), 118-127.

[16] Lepowsky J., Li H., Introduction to vertex operator algebras and their representations, Progress in Mathematics, Vol. 227, Birkhäuser Boston, Inc., Boston, MA, 2004.

[17] Li H.S., Symmetric invariant bilinear forms on vertex operator algebras, J. Pure Appl. Algebra 96 (1994), 279-297.

[18] Liu D., Zhu L., Classification of Harish-Chandra modules over the $W$-algebra $W(2,2)$, arXiv:0801.2601.

[19] Radobolja G., Subsingular vectors in Verma modules, and tensor product of weight modules over the twisted Heisenberg-Virasoro algebra and $W(2,2)$ algebra, J. Math. Phys. 54 (2013), 071701, 24 pages, arXiv:1302.0801.

[20] Zhang W., Dong C., $W$-algebra $W(2,2)$ and the vertex operator algebra $L\left(\frac{1}{2}, 0\right) \otimes L\left(\frac{1}{2}, 0\right)$, Comm. Math. Phys. 285 (2009), 991-1004, arXiv:0711.4624. 